\title{
Abnormal Action Potential Duration Restitution Property in the Right Ventricular Outflow Tract in Brugada Syndrome
}

\author{
Sonoko Ashino, MD; Ichiro Watanabe, MD; Masayoshi Kofune, MD; Koichi Nagashima, MD; \\ Kimie Ohkubo, MD; Yasuo Okumura, MD; Toshiko Nakai, MD; \\ Yuji Kasamaki, MD; Atsushi Hirayama, MD
}

\begin{abstract}
Background: Although patients with Brugada syndrome (BS) are at risk of ventricular fibrillation (VF) and ensuing death, the action potential duration (APD) restitution properties of the right ventricular outflow tract (RVOT) in patients with BS remain undetermined.
\end{abstract}

\begin{abstract}
Methods and Results: Endocardial monophasic action potentials (MAPs) were obtained from 16 patients with BS and 17 control patients. MAPs were recorded from the RVOT in all patients. The MAP duration at $90 \%$ repolarization (MAPD90), effective refractory period (ERP), and maximum slope of the APD restitution curve were obtained. VF was induced with up to 3 extrastimuli from the RV apex or RVOT. There was no difference in MAPDgo between the 2 groups, but the ERP was significantly shorter in patients with BS than in control patients $(210.7 \pm 10.5$ vs 223.8 $\pm 13.4 \mathrm{~ms}, P=0.008)$. MAPD at the shortest diastolic interval was significantly shorter in patients with $B S$ than in control patients $(149.9 \pm 19.9$ vs $179.8 \pm 13.7 \mathrm{~ms}, \mathrm{P}<0.001)$. The maximum slope of the APD restitution curve was steeper in patients with $B S$ than in control patients $(2.90 \pm 1.29$ vs $1.38 \pm 0.41, P<0.001)$.
\end{abstract}

Conclusions: The shorter ERP, shorter MAPD at the shortest diastolic interval and steeply sloped APD restitution curve in the RVOT appear to be related to the inducibility of VF in patients with BS. (Circ J 2010; 74: 664-670) Key Words: Action potential duration restitution; Brugada syndrome; Monophasic action potential; Ventricular
fibrillation; Ventricular refractory period

B rugada syndrome (BS) is characterized electrocardiographically by a right bundle branch block pattern and ST-segment elevation in precordial leads $\mathrm{V}_{1-3}$, and also by a propensity for sudden cardiac death because of ventricular fibrillation (VF). ${ }^{1}$ Several studies have linked BS to 1 or more gene mutations; $;^{2-4}$ however, the mechanisms of arrhythmogenesis remain controversial. Animal studies suggest that the ECG "Brugada sign" reflects loss of the action potential dome in the epicardium but not in the endocardium, allowing extra beats (from phase 2 reentry) to interact with transmembrane repolarization dispersion to initiate reentry (repolarization disorder) and/or conduction delay in the right ventricular (RV) outflow tract $(\mathrm{OT}) .^{5-7}$ However, the factors that cause degeneration into $\mathrm{VF}$ and continuation of the fibrillatory activities, perhaps providing the background for inducible sustained VF during electrophysiologic study (EPS), have not been elucidated. The electrical restitution property of the myocardium has been shown to play an important role in determining the susceptibility of the heart to fibrillation. Evidence from experimental ${ }^{8-10}$ and clinical studies ${ }^{11,12}$ indicates that the maximum slope of the ventricular action potential duration (APD) restitution curve, which portrays the relation between the local APD and the preceding diastolic interval, reflects the propensity for VF. If the slope of the restitution function at a short diastolic interval exceeds unity, small changes in the diastolic interval can produce large fluctuations in the APD and refractoriness, ${ }^{9,13}$ which may lead to functional gradients in repolarization that may promote conduction block and the wavebreak of re-entrant wavefronts. ${ }^{14}$ Two groups of investigators have documented that the maximum slope of the APR restitution curve in the RV apex (RVA) is greater than that in the RVOT. ${ }^{15,16}$ However, we showed repetitive ventricular tachycardia (VT) originating from the RVOT and degenerating into VF in a majority of patients with BS. ${ }^{17}$ We also reported a case in which monophasic action potentials (MAPs) recorded from the RVOT at a basic cycle length (CL) of $400 \mathrm{~ms}$ showed MAP alternans, and VF was induced only when extrastimuli were applied after a decrease in the MAP duration (MAPD) of the alternans. ${ }^{18}$ Thus we hypothesized that the RVOT contributes to

Received November 11, 2009; revised manuscript received December 25, 2009; accepted January 5, 2010; released online February 27,

2010 Time for primary review: 32 days

Division of Cardiology, Department of Medicine, Nihon University School of Medicine, Tokyo, Japan

Mailing address: Ichiro Watanabe, MD, Division of Cardiology, Department of Medicine, Nihon University School of Medicine, 30-1

Oyaguchi-kami, Itabashi-ku, Tokyo 173-8610, Japan. E-mail: iwatanab@med.nihon-u.ac.jp

ISSN-1346-9843 doi:10.1253/circj.CJ-09-0872

All rights are reserved to the Japanese Circulation Society. For permissions, please e-mail: cj@j-circ.or.jp 
the inducibility of VF in patients with BS undergoing programmed ventricular stimulation. To examine this hypothesis, we recorded MAPs from the RVOT to calculate the repolarization restitution slope during programmed ventricular stimulation.

\section{Methods}

\section{Subjects and Clinical Data Collection}

BS Patients Sixteen patients diagnosed with BS between 2004 and 2008 comprised the BS group. Clinical data, including age at diagnosis, sex, family history of sudden death, documented VF, episodes of syncope, and implantable cardioverter-defibrillator (ICD) implantation, were obtained for all patients. Structural heart disease was ruled out in each case on the basis of the patient's clinical history and by extensive evaluation, including non-invasive and invasive procedures (echocardiography, coronary angiography, and left and right ventriculography). A family history of sudden death was defined as sudden death of unknown cause in a family member at less than 50 years of age. Thirteen patients showed a typical type 1 Brugada pattern as previously defined. ${ }^{19}$ In the remaining 3 patients with a type $2(n=1)$ or $3(n=2)$ Brugada pattern, $1 \mathrm{mg} / \mathrm{kg}$ of pilsicainide (a pure sodium channel blocker) was administered intravenously for $10 \mathrm{~min}$ with continuous monitoring, and it was confirmed that the Brugada pattern changed to type 1 . Fourteen of the 16 patients provided informed consent for investigation of SCN5A gene mutation, but no mutation was found.

Control Patients Seventeen age-matched patients who underwent catheter ablation for tachyarrhythmia but did not have a Brugada-type ECG comprised the control group. Structural heart disease was ruled out in all cases on the basis of the patient's clinical history and by extensive evaluation, including non-invasive and invasive procedures (echocardiography, coronary angiography, and left and right ventriculography).

This single-center study complied with the guidelines of the Declaration of Helsinki and was approved by the institutional ethics committee.

\section{EPS}

Comprehensive EPS was performed in all BS and control patients after written informed consent was given. The EPS was conducted with subjects in a fasting, drug-free, and nonsedated state. After access to the right femoral vein was obtained at 2 sites, 2 steerable quadripolar catheters $(6 \mathrm{~F})$ with an interelectrode distance of 2-5-2 mm (Biosense-Webster, Diamond Bar, CA, USA) were positioned in the RV. Endocardial potentials were filtered to recording frequencies of $30-500 \mathrm{~Hz}$ and recorded on a BARD computer system. Programmed electrical stimulation from the RVA and RVOT was performed at twice the diastolic threshold strength and a pulse of 2-ms duration with a pulse generator (BC-02, Fukuda Denshi Co, Tokyo, Japan). An $\mathrm{S}_{1}-\mathrm{S}_{2}$ interval was applied after 8 beats of drive pacing $\left(\mathrm{S}_{1}\right)$ at basic CLs of $600 \mathrm{~ms}$ and $400 \mathrm{~ms}$. The $\mathrm{S}_{1}-\mathrm{S}_{2}$ interval was decreased in 10-ms steps until the effective refractory period (ERP) of the RV was reached. When $\mathrm{VF}$ that lasted $>10 \mathrm{~s}$ and required DC shock was not induced with a single premature beat, up to 3 extrastimuli $\left(\mathrm{S}_{1} \mathrm{~S}_{2}\right.$ until the ERP was reached, $\mathrm{S}_{2} \mathrm{~S}_{3}$ and $\mathrm{S}_{3} \mathrm{~S}_{4}$ to $180 \mathrm{~ms}$ ) were delivered. Patients with polymorphic VT or VF that terminated spontaneously within $10 \mathrm{~s}$ were not included in this study.

A 7F MAP recording/pacing catheter (Franz ${ }^{\circledR}$ catheter, EP Technologies Inc, San Jose, CA, USA) was inserted through the right femoral vein and positioned in the RVOT at $20 \mathrm{~min}$ after DC shock for patients with BS or after successful ablation in control patients. MAP signals were amplified at a filter setting of $0.05-500 \mathrm{~Hz}$. Ventricular pacing was performed from the proximal electrode pair of the Franz catheter at twice the diastolic threshold strength and a pulse of 2-ms duration. The MAPD was measured as the interval along a line horizontal to the diastolic baseline from the steepest part of the MAP upstroke to the level of $90 \%$ repolarization (MAPD90). ${ }^{20}$

\section{Construction of the APD Restitution Curve}

Restitution of the MAPD90 was determined by using a single $S_{2}$ delivered after every $12^{\text {th }} S_{1}$ at a basic CL of $600 \mathrm{~ms}$. The $S_{1} S_{1}$ interval was started at $600 \mathrm{~ms}$ and was decreased in 20-ms steps until an $\mathrm{S}_{1} \mathrm{~S}_{2}$ of $400 \mathrm{~ms}$ was reached. It was decreased in 10-ms steps until an $\mathrm{S}_{1} \mathrm{~S}_{2}$ of $300 \mathrm{~ms}$ was reached, and then in 5-ms steps until ventricular capture failed. After each $\mathrm{S}_{2}, \mathrm{~S}_{1}$ pacing was interrupted for $1.5 \mathrm{~s}$. No VT/VF was induced by single ventricular extrastimulus from the MAP recording/pacing catheter. The APD restitution curve was constructed by plotting the MAPD 90 of $\mathrm{S}_{2}$ vs the preceding diastolic interval, which was obtained by subtracting the MAPD 90 of the $11^{\text {th }} \mathrm{S}_{1}$ from the $\mathrm{S}_{1} \mathrm{~S}_{2}$ interval and adding the latency (stimulus-tissue delay) after $\mathrm{S}_{2}$. The time course of restitution obtained by $\mathrm{S}_{1} \mathrm{~S}_{2}$ was fitted by using the monoexponential equation with the use of Origin 7.0 software (OriginLab Corp, Northampton, MA, USA), and the maximum slope of the APD restitution curve was obtained by the maximum value of the first derivative of the mono-exponential equation fitting curve. ${ }^{15,21,22}$

\section{Statistical Analysis}

Continuous data are presented as mean \pm SD. Differences in continuous clinical variables were analyzed by two-tailed t-test. Differences in repolarization and conduction parameters were analyzed by Mann-Whiney U test. A P value $<0.05$ was considered statistically significant. StatView 5.0 software (SAS Institute, Cary, NC, USA) was used for analysis.

\section{Results}

\section{Patient Characteristics}

Left ventricular ejection fraction did not differ between the BS and control patients $(67.8 \pm 5.9 \%$ vs $67.6 \pm 6.4 \%$, respectively) nor did the left atrial diameter $(31.3 \pm 5.1 \mathrm{~mm}$ vs $31.2 \pm$ $5.0 \mathrm{~mm}$, respectively). The BS patients were all probands. None of the patients in this study were members of the same family. Aborted sudden cardiac death occurred in 1 patient, episodes of syncope occurred in 1 patient, and another 2 patients had a family history of unexplained sudden death (Table 1). A spontaneous type 1 ECG pattern was observed in 13 of the 16 patients with BS. The control group consisted of 4 patients with concealed Wolf-Parkinson-White syndrome, 11 patients with atrioventricular nodal reentrant tachycardia, and 2 patients with premature ventricular contraction of RVOT origin.

\section{EPS}

A type 1 ECG pattern was observed in 13 of the 16 patients with BS during the EPS. There was no significant difference between the groups in terms of the AH interval (103.3 \pm $25.0 \mathrm{~ms}$ in BS patients vs $93.0 \pm 12.6 \mathrm{~ms}$ in control patients, $\mathrm{P}=0.358)$ or the $\mathrm{HV}$ interval $(48.4 \pm 11.5 \mathrm{~ms}$ in $\mathrm{BS}$ patients vs $43.1 \pm 5.2 \mathrm{~ms}$ in control patients, $\mathrm{P}=0.242$ ) (Table 2). Oneto-one conduction of the atrioventricular node did not differ 
Table 1. Clinical Characteristics Per Study Group

\begin{tabular}{lccc} 
& Brugada $(\mathbf{n = 1 6 )}$ & Control $(\mathbf{n}=\mathbf{1 7})$ & P value \\
Clinical data & & & 0.497 \\
Age (years) & $51.2 \pm 13.2$ & $54.2 \pm 11.9$ & $<0.001$ \\
Sex ratio (M/F) & $16 / 0$ & $8 / 9$ & \\
Spontaneous type 1 ECG & 13 & 0 & \\
Family history of sudden death & 2 & 0 & \\
Syncopal episode & 1 & 0 & 0.955 \\
Documented VF & 1 & 0 & 0.926 \\
Echocardiographic data & & & \\
Left atrial diameter (mm) & $31.3 \pm 5.1$ & $67.2 \pm 5.0$ & \\
LVEF $(\%)$ & $67.8 \pm 5.9$ & & \\
\hline
\end{tabular}

Number of patients is shown unless otherwise indicated.

VF, ventricular fibrillation; LVEF, left ventricular ejection fraction.

Table 2. Electrophysiologic Characteristics Per Study Group

\begin{tabular}{lccc} 
& Brugada $(\mathbf{n}=\mathbf{1 6})$ & Control $(\mathbf{n}=\mathbf{1 7})$ & P value \\
AH interval $(\mathrm{ms})$ & $103.3 \pm 25.0$ & $93.0 \pm 12.6$ & 0.358 \\
$\mathrm{HV}$ interval $(\mathrm{ms})$ & $48.4 \pm 11.5$ & $43.1 \pm 5.2$ & 0.242 \\
Wenckebach cycle length (ms) & $409.6 \pm 51.8$ & $381.5 \pm 68.3$ & 0.320 \\
ERP of the RVOT (ms) & $211.9 \pm 11.2$ & $233.8 \pm 13.4$ & 0.008 \\
MAPD90 of the RVOT (ms) & $226.6 \pm 15.5$ & $233.9 \pm 15.9$ & 0.192 \\
MAPD90 at the shortest DI (ms) & $149.9 \pm 19.9$ & $179.8 \pm 13.7$ & $<0.001$ \\
Maximum MAPDgo slope & $2.90 \pm 1.28$ & $1.38 \pm 0.91$ & $<0.001$ \\
VF induction during EP study & $16 / 16$ & $\mathrm{NA}$ & \\
\hline
\end{tabular}

ERP, effective refractory period; RVOT, right ventricular outflow tract; MAPD $90,90 \%$ repolarization of the monophasic action potential; DI, diastolic interval; NA, not appropriate. Other abbrevition see in Table 1.

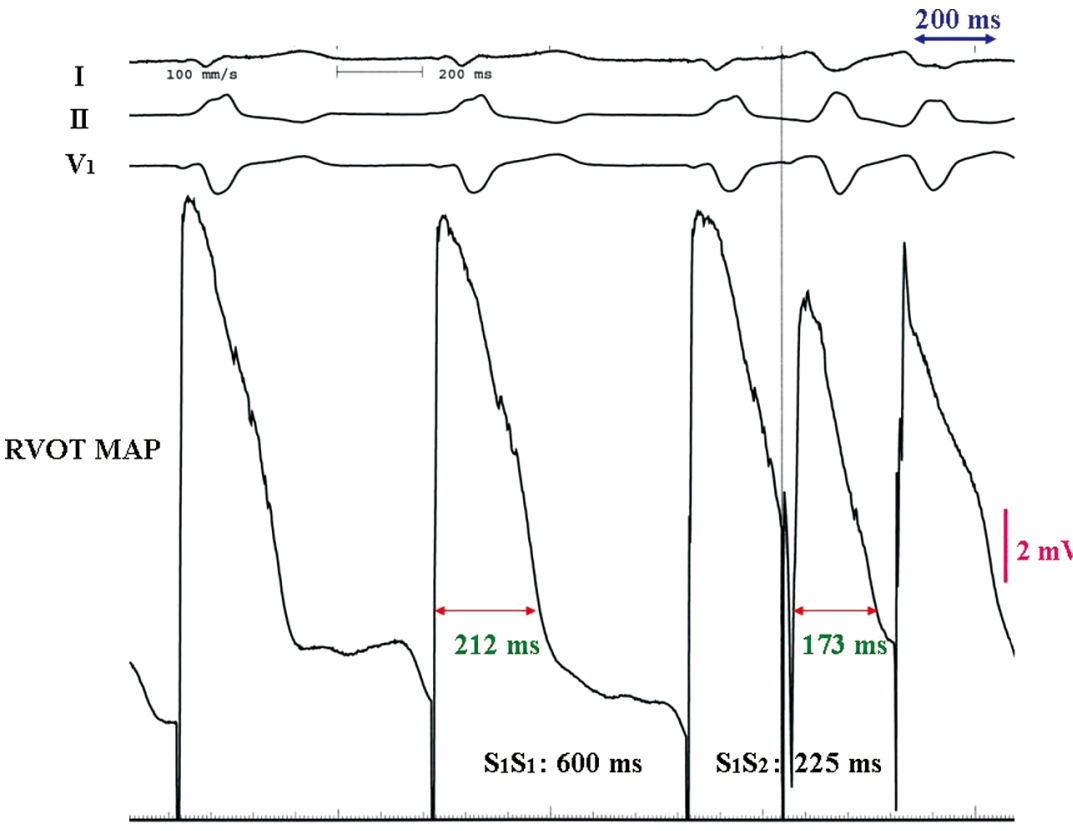

DI: $+37 \mathrm{~ms}$
Figure 1. Right ventricular MAP recordings at the shortest $\mathrm{DI}$ in a control patient. Effective refractory period of the RVOT of this patient was $220 \mathrm{~ms}$. MAP, monophasic action potential; RVOT, right ventricular outflow tract; DI, diastolic interval. significantly between the BS and control patients (409.6 \pm $51.8 \mathrm{~ms}$ vs $381.5 \pm 68.3 \mathrm{~ms}, \mathrm{P}=0.320$ ). The ERP-RVOT at a basic CL of $600 \mathrm{~ms}$ was significantly shorter in the BS group than in the control group $(211.9 \pm 11.2 \mathrm{~ms}$ vs $223.8 \pm 13.4 \mathrm{~ms}$, $\mathrm{P}=0.008$ ), but there was no statistically significant difference between the 2 groups in the RVOT MAPD $90(226.6 \pm 15.5 \mathrm{~ms}$ vs $233.9 \pm 15.9 \mathrm{~ms})$. The RVOT MAPD 90 at the shortest diastolic interval was significantly shorter in the BS group (Figures 2,4) than in the control group (Figures 1,3) (149.9 \pm $19.9 \mathrm{~ms}$ vs $179.8 \pm 13.7 \mathrm{~ms}, \mathrm{P}<0.001)$. The maximum slope of 


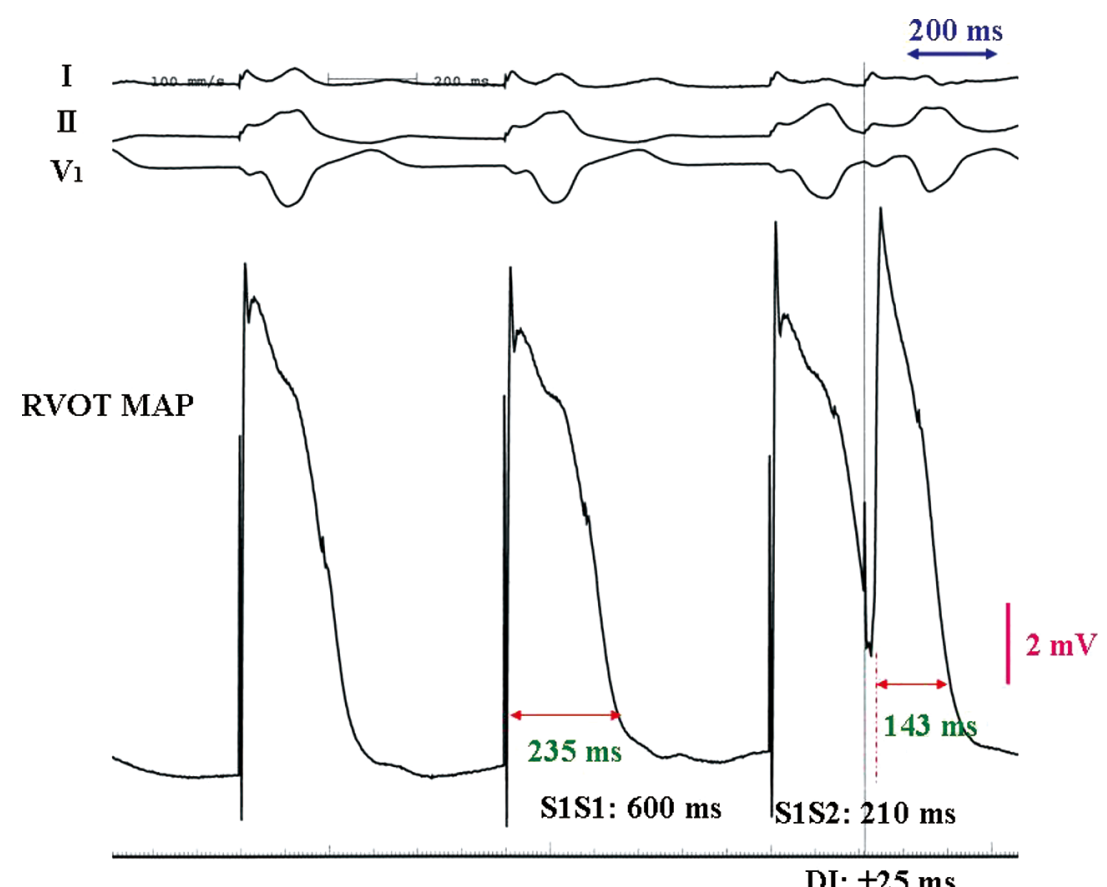

DI: $+25 \mathrm{~ms}$
Figure 2. Right ventricular MAP recordings at the shortest DI in a patient with Brugada syndrome. Effective refractory period of the RVOT of this patient was $205 \mathrm{~ms}$. RVOT, right ventricular outflow tract, MAP, monophasic action potential; DI, diastolic interval.

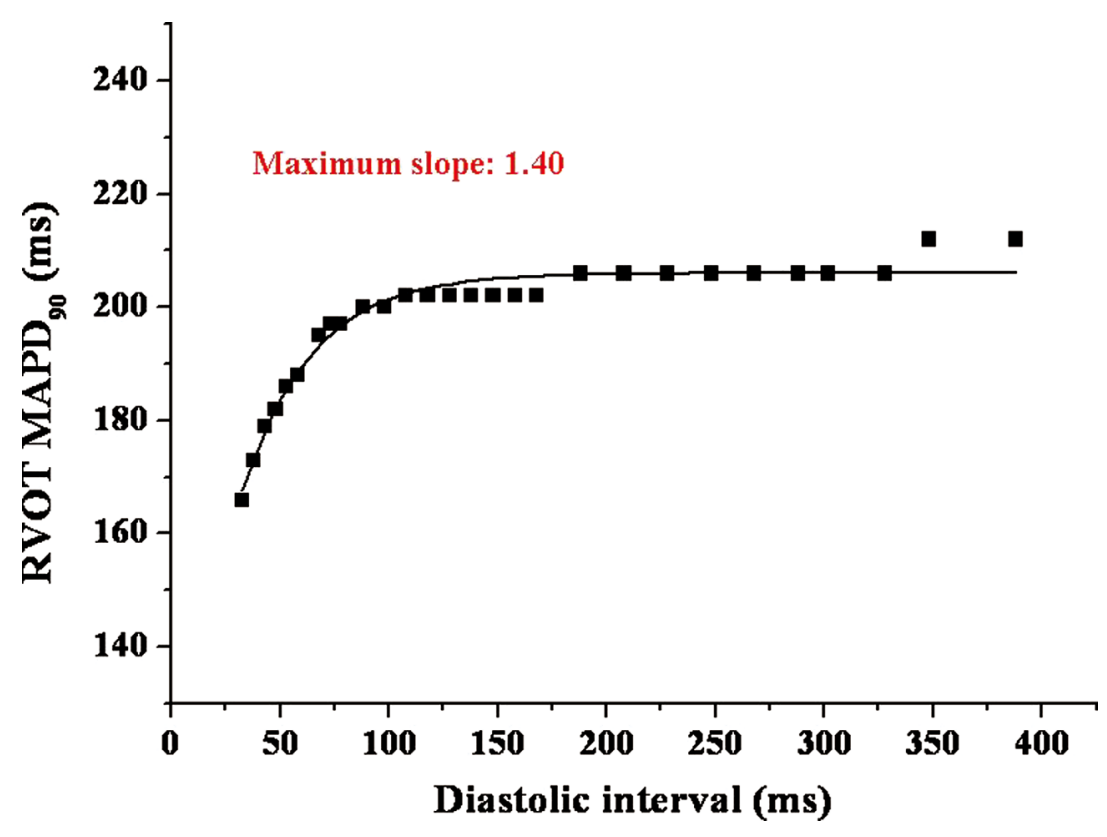

Figure 3. Electrical restitution curve at the RVOT in the control patient shown in Figure 1. According to a single linear plot, the maximum slope was 1.4. RVOT, right ventricular outflow tract; MAPD9o, monophasic action potential duration at $90 \%$ repolarization.

the RVOT MAPD90 restitution curve was significantly greater in the BS group than in the control group $(2.90 \pm 1.28[1.0$ 4.6] vs 1.38 \pm 0.41 [1.0-2.4], $\mathrm{P}<0.001$ ) (Figures 3,4; Table 2). Polymorphic VT or VF lasting $>10$ s requiring DC shock was induced in all 16 patients with BS (Table 3). Because all patients had spontaneous type $1 \mathrm{ECG}$ and/or syncopal episode, family history of sudden death, documented VF, and inducible VF, we recommended implanting an ICD, which 12 of the 16 patients $(75.0 \%)$ did receive; 4 patients refused. Patients with BS were followed-up in the outpatient clinic every 6 months for 40.6 \pm 19.2 months (11-65 months).
No sudden cardiac death or appropriate ICD shock was noted during the follow-up period.

\section{Discussion}

Repolarization Properties and the Inducibility of Ventricular Arrhythmias

The present study compared the ERP and MAPD 90, MAPD 90 at the shortest diastolic interval, and steepness of the maximum APD restitution slope of the RVOT between BS patients and control patients. We showed that the ERP-RVOT and 


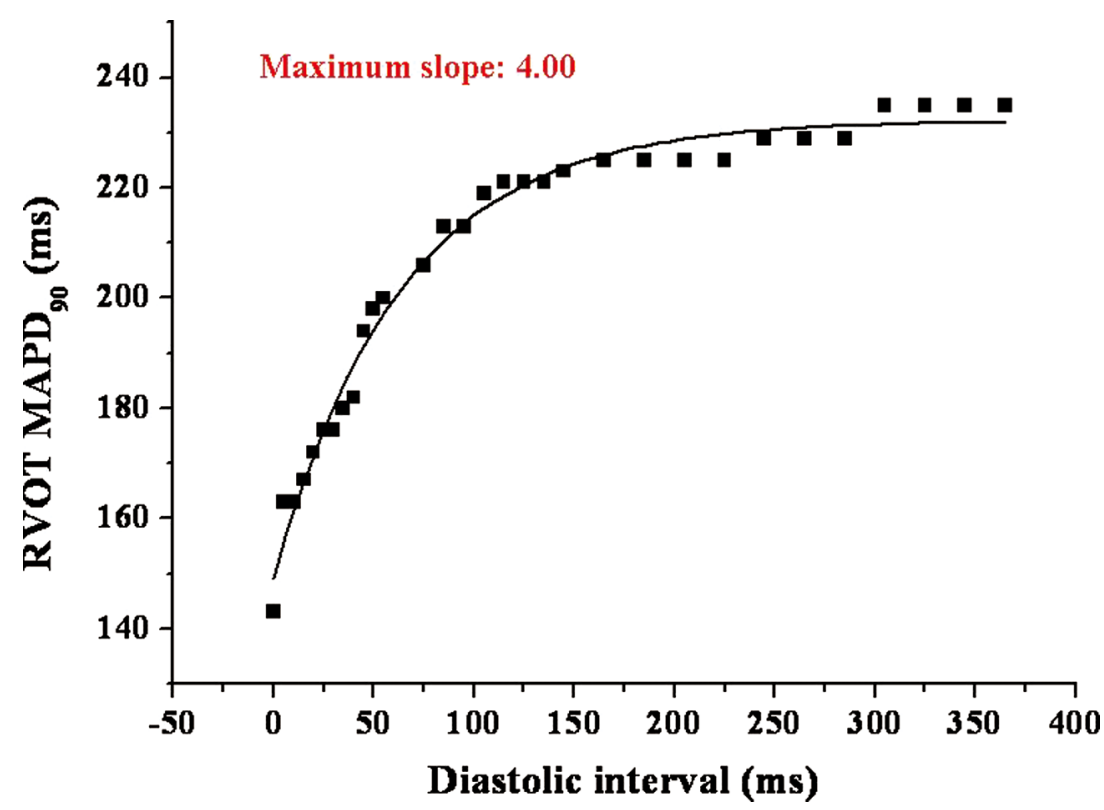

Figure 4. Electrical restitution curve at the RVOT in the Brugada syndrome patient shown in Figure 2. According to a single linear plot, the maximum slope was 4.0. RVOT, right ventricular outflow tract; MAPDgo, monophasic action potential duration at $90 \%$ repolarization.

\begin{tabular}{|c|c|}
\hline Patient no. & VF induction (ms) \\
\hline 1 & RVOT: 600/230/220 \\
\hline 2 & RVOT: 600/230/200/200 \\
\hline 3 & RVOT: 400/250/190 \\
\hline 4 & RVOT: 600/230/200 \\
\hline 5 & RVOT: 600/250/230 \\
\hline 6 & RVOT: 600/230/220 \\
\hline 7 & RVOT: 400/220/210 \\
\hline 8 & RVOT: 400/270/270/200 \\
\hline 9 & RVOT: 600/250/200 \\
\hline 10 & RVA: 600/230/210 \\
\hline 11 & RVOT: 400/220/200 \\
\hline 12 & RVA: 400/230/200 \\
\hline 13 & RVOT: 600/270/250 \\
\hline 14 & RVA: 600/270/200 \\
\hline 15 & RVA: 600/220/220 \\
\hline 16 & RVA: 400/210/200 \\
\hline
\end{tabular}

RVA, right ventricular apex. Other abbrevitions see in Tables 1,2.

MAPD $90_{90}$ at the shortest diastolic interval were significantly shorter, and the maximum RVOT APD restitution slope was significantly steeper, in patients with BS. Recent studies have shown that the maximum slope is steeper in patients with inducible $\mathrm{VT}^{21,23}$ and that these patients had a greater risk of sudden cardiac death than did those without. ${ }^{23}$ Furthermore, recent studies have examined the repolarization restitution properties in patients with BS. Narayan et al reported that the RV MAPD restitution slope was steeper in the RVA than in the RVOT in a patient with inducible $\mathrm{VF},{ }^{16}$ and Hayashi et al reported that the maximum activation recovery interval restitution slope in the RVA, but not the RVOT, was steeper in BS patients with inducible VF than in those without. ${ }^{14}$ In a reported case of short-coupled torsades de pointes, the patient's MAPD was significantly shorter at the RVA than at the RVOT, and the maximum slope of the MAPD restitution curve was much steeper at the RVA than at the RVOT. ${ }^{22}$

Previously, we recorded low-amplitude fragmented and delayed potentials at the RVOT in BS patients with inducible $\mathrm{VF},{ }^{23}$ and we found that a majority of patients with BS showed repetitive VT originating from the RVOT that degenerated into VF. ${ }^{17}$ Moreover Park et al reported that the maximum slope of the APD restitution curve in BS patients with inducible VF was 2.1 at the RVOT and significantly greater than that at the RVA (1.1). ${ }^{24}$ To our knowledge, we provide the first description of the detailed APD restitution properties in BS. Theoretically, if the APD restitution slope exceeds unity, the wavelength oscillations self-amplify and the diastolic interval becomes too short for the wave to propagate, resulting in conduction block and wavebreak. ${ }^{13,14}$ In the present study, the maximum APD restitution slope was shown to be steeper in BS patients with inducible VF, and a slope $>1$ in the RVOT was observed in $94.4 \%$ of the patients. Abrupt shortening of the APD in premature beats close to the refractory period is the primary reason for a steep repolarization restitution slope. In the earliest premature responses, a less-negative initial membrane voltage, resulting from incomplete sodium channel activation diminishes the following L-type calcium activity, which is responsible for the action potential plateau, and foreshortens the APD. ${ }^{25}$ In BS, mutations in the cardiac sodium channel gene SCN5A have been found and shown to result in a loss of the channel function, ${ }^{2}$ and several experimental studies ${ }^{26,27}$ have revealed a significantly more delayed "slow" inactivation in the cells with a mutant SCN5A channel than in those without. In BS, in the case of premature beats preceded by very short diastolic intervals, the degree of curtailment of the action potential plateau would be related to the severity of reduced sodium channel activity. Another possibility for the steep repolarization slope in BS is the excessive activation of the transient outward current. We reported that intravenous administration of quinidine inhibited the induction of VF by programmed ventricular stimulation, and that the mechanism was related to the increase in the shortest APD and decrease in the restitution slope after quinidine administration. ${ }^{28}$ 


\section{Mechanism of VF in BS}

Experimental BS models have shown that a local re-excitation called phase 2 reentry captures the vulnerable windows and triggers a circus movement reentry. ${ }^{5,6,29} \mathrm{We}$ assessed the repolarization restitution property and showed that the maximum restitution slope in the RVOT of BS patients with inducible VF is significantly steeper than that of control patients, which indicates that the RVOT region may play an important role in the degeneration into sustained VF in patients with BS. Previous studies have also revealed the importance of the RVOT in the development of VF in BS, based on the presence of delayed potentials in the RVOT, ${ }^{30}$ the presence of initial repetitive VT originating from the RVOT before degeneration into $\mathrm{VF},{ }^{17}$ and development of spontaneous premature contractions triggering VF mainly in the RVOT. ${ }^{31}$ Transmural dispersion of repolarization in the RVOT has been shown to contribute to initial repetitive excitation during VT in experimental BS models.,5,29 Previous studies have shown the presence of spatial heterogeneity of APD restitution properties in patients with BS and a shortcoupled variant of torsades de pointes..$^{15,16,22}$ However, a previous study in the explanted Langendorff-perfused heart from a patient with $\mathrm{BS}$ at heart transplantation failed to show steep APD restitution and the importance of slow conduction in the right ventricle. ${ }^{32}$ In vivo human heart studies also have shown broad restitution of propagation in patients with BS. ${ }^{15,16,33,34}$ A recent report showed that the critical parameter for differentiating between the occurrence of reentry and the mere occurrence of a line of activation block between 2 myocardial regions (and no reentry) was not the magnitude of the premature activation wave at the distal side of the premature beat proximal to the line of block, but rather the restitution characteristics of the tissue with shorter action potential, in combination with the time of arrival of the premature wavefront at the distal end side of the line of block, determines the occurrence of reentry. ${ }^{35}$ We observed a case of BS in which VF was induced by a single premature ventricular stimulus from a MAP catheter (Figure 5). Following a very short MAP induced by an extrastimulus, a premature ventricular beat occurred, preceded by an MAP from the same recording site, leading to $\mathrm{VF}$.

\section{Methodological Consideration for the Steepness of the Repolarization Restitution Slope}

The value of the maximal slope of the repolarization restitution curve in clinical studies using MAPD varies. Pak et al showed that the mean maximum slopes of the RVOT and RVA in control patients measured at a basic CL of $600 \mathrm{~ms}$ were $1.9 \pm 1.1$ and $1.7 \pm 1.1$, respectively. ${ }^{21}$ Yamazaki et al showed that the mean maximum slopes in the RVOT and RVA in control patients measured at a basic CL of $600 \mathrm{~ms}$ were $1.26 \pm 0.66$ and $1.27 \pm 0.51$, respectively, ${ }^{22}$ comparable to values shown in our control patients. Pak et al reported that the mean maximum slopes of the RVOT and RVA in inducible VT group patients measured at a basic CL of $600 \mathrm{~ms}$ were $3.7 \pm 2.1$ and $2.3 \pm 2.7$, respectively. ${ }^{21}$ Park et al also reported that the mean maximum slopes of the RVOT and RVA in BS patients with inducible VF were 2.1 and 1.1, respectively, ${ }^{24}$ also comparable to values shown in our BS patients with inducible VF.

\section{Study Limitations}

Our study might have been limited by the fact that the MAP catheter records electrical activity from multiple cells in close proximity to the recording electrodes. ${ }^{20,36}$ The amplitude of

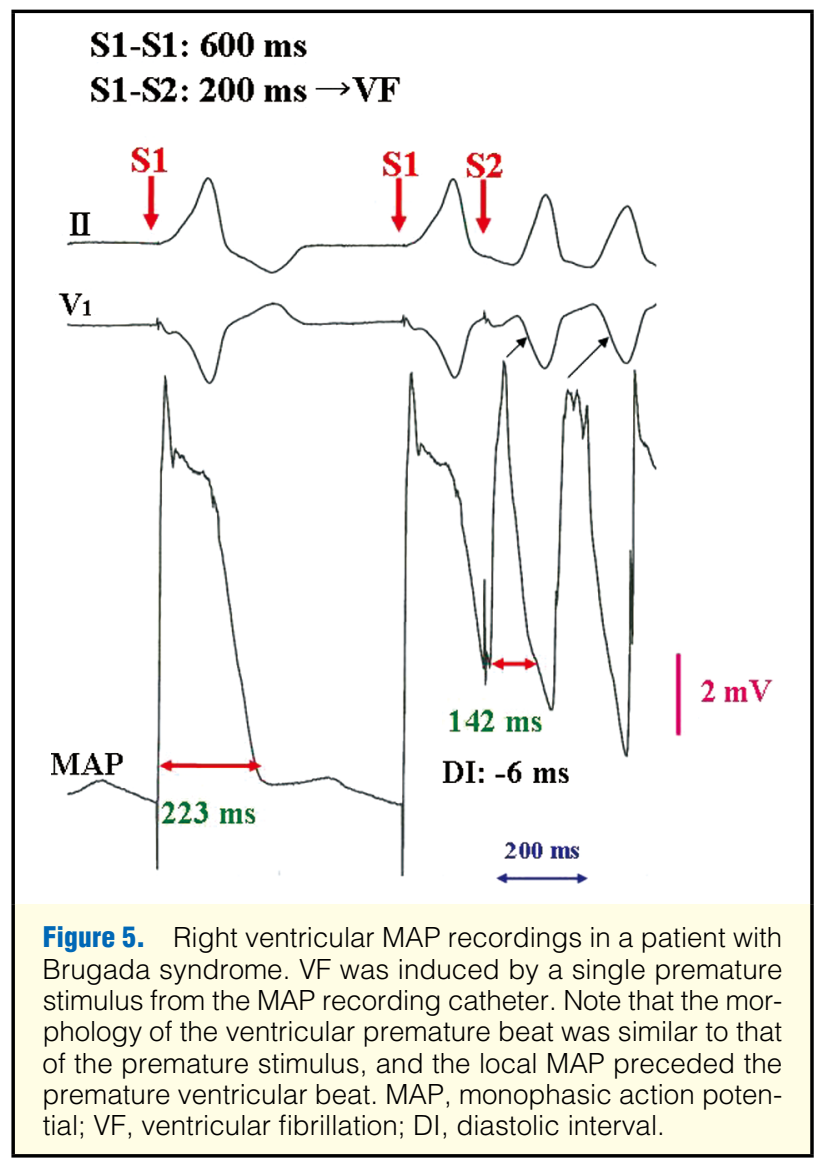

the MAP recording declines gradually over the study. ${ }^{37}$ However, the APD restitution curve recorded with the MAP electrode closely resembles the APD restitution curve obtained by the microelectrode technique. ${ }^{38} \mathrm{~A}$ second limitation is that only APD restitution from the RVOT was measured, making determination of spatial heterogeneity of APD restitution inapplicable. Third, comparison of APD restitution between BS patients with and without documented VT/VF and between those with and without inducible VT/VF was not done. Fourth, restitution of propagation was not measured in the present study. Finally, there was a significant difference in the sex ratio between the BS group and the control group in the present study, but there was no significant difference in the ERP-RVOT (219.4 $\pm 14.5 \mathrm{~ms}$ vs $227.8 \pm 11.8 \mathrm{~ms}, \mathrm{P}=0.173)$, MAPD90-RVOT (227.4 \pm 4.6 vs $239.7 \pm 5.5 \mathrm{~ms}, \mathrm{P}=0.101)$, shortest MAPD90-RVOT (175.8 \pm 4.4 vs $183.4 \pm 4.8 \mathrm{~ms}, \mathrm{P}=0.308)$, or maximum slope of the MAPD90-RVOT restitution curve $(1.19 \pm 0.006$ vs $1.544 \pm 0.166, P=0.115)$ between males and females in the control group. Recent studies have shown that propagation restitution, in addition to steep APD restitution, is important in determining myocardial electrical instability. ${ }^{16,39}$ Further studies should use epicardial as well as endocardial mapping to better define the role of repolarization, conduction, and transmural dispersion in BS-related arrhythmogenesis.

\section{Conclusions}

Results of the present study suggest that the repolarization restitution property may relate to the propensity for VF in BS. The maximum APD restitution slope in the RVOT was 
steeper in BS patients with inducible VF than in control patients. Further studies are needed to record MAPs from multiple sites within the RV and to simultaneously measure propagation restitution to better define the role of repolarization, spatial dispersion of repolarization, and conduction in BS-related arrhythmogenesis.

\section{Disclosures}

The authors have no conflict of interests to disclose. The study was supported by departmental resources only.

\section{References}

1. Brugada P, Brugada J. Right bundle branch block, persistent ST segment elevation and sudden cardiac death: A distinct clinical and electrocardiographic syndrome: A multicenter report. J Am Coll Cardiol 1992; 20: $1391-1396$.

2. Chen Q, Kirsch GE, Zhang D, Brugada R, Brugada J, Brugada P, et al. Genetic basis and molecular mechanism for idiopathic ventricular fibrillation. Nature 1998; 392: 293-296.

3. Antzelevitch C, Pollevick GD, Corderio JM, Casis O, Sanguinetti MC, Aizawa Y, et al. Loss-of-function mutations in the cardiac calcium channel underlie a new clinical entity characterized by STsegment elevation, short QT intervals, and sudden cardiac death. Circulation 2007; 115: $442-449$.

4. London B, Michalec M, Mehdi H, Zhu X, Kerchner L, Sanyal S, et al. Mutation in glycerol-3-phosphate dehydrogenase 1-like gene (GPD1-L) decreases cardiac $\mathrm{Na}^{+}$current and causes inherited arrhythmias. Circulation 2007; 116: 2260-2268.

5. Yan GX, Antzelevitch C. Cellular basis for the Brugada syndrome and other mechanisms of arrhythmogenesis associated with STsegment elevation. Circulation 1999; 100: 1660-1666.

6. Aiba T, Shimizu W, Hidaka I, Uemura K, Noda T, Zheng C, et al. Cellular basis for trigger and maintenance of ventricular fibrillation in the Brugada syndrome model: High-resolution optical mapping study. J Am Coll Cardiol 2006; 47: 2074-2085.

7. Meregalli PG, Wilde AA, Tan HL. Pathophysiological mechanisms of Brugada syndrome: Depolarization disorder, repolarization disorder, or more? Cardiovasc Res 2005; 67: 367-378.

8. Omichi C, Zhou S, Lee MH, Naik A, Chang CM, Garfinkel A, et al. Effects of amiodarone on wave front dynamics during ventricular fibrillation in isolated swine right ventricle. Am J Physiol Heart Circ Physiol 2002; 282: H1063-H1070.

9. Riccio ML, Koller ML, Gilmour RF Jr. Electrical restitution and spatiotemporal organization during ventricular fibrillation. Circ Res 1999; 84: $955-963$.

10. Wu TJ, Lin SF, Weiss JN, Ting CT, Chen PS. Two types of ventricular fibrillation in isolated rabbit hearts: Importance of excitability and action potential duration restitution. Circulation 2002; 106: $1859-1866$.

11. Koller ML, Maier SK, Gelzer AR, Bauer WR, Meesmann M, Gilmour RF. Altered dynamics of action potential restitution and alternans in humans with structural heart disease. Circulation 2005; 112: $1542-1548$.

12. Taggart P, Sutton P, Chalabi Z, Boyett MR, Simon R, Elliott D, et al. Effect of adrenergic stimulation on action potential duration restitution in humans. Circulation 2003; 107: 285-289.

13. Qu Z, Weiss JN, Garfinkel A. Cardiac electrical restitution properties and stability of reentrant spiral waves: A simulation study. Am J Cardiol 1999; 276: H269-H283.

14. Karma A. Electrical alternans and spiral wave breakup in cardiac tissue. Chaos 1994; 4: 461-472.

15. Hayashi M, Takatsuki S, Maison-Blanche P, Messali A, Haggui A, Milliez $\mathrm{P}$, et al. Ventricular repolarization restitution properties in patients exhibiting type 1 Brugada electrogram with and without inducible ventricular fibrillation. J Am Coll Cardiol 2008; 51: $1162-1168$.

16. Narayan SM, Kim J, Tate C, Berman BJ. Steep restitution of ventricular action potential duration and conduction slowing in human Brugada syndrome. Heart Rhythm 2007; 4: 1087-1089.

17. Ohkubo K, Watanabe I, Okumura Y, Ashino S, Kofune M, Ohta M, et al. Surface ECG characteristics of ventricular tachycardias before degenerating into ventricular fibrillation in patients with Brugadatype ECG. Int Heart J 2009; 50: 477-487.

18. Kofune M, Watanabe I, Ashino S, Ohkuno K, Okumura Y, Kofune $\mathrm{T}$, et al. Action potential alternans in the right ventricular outflow tract in a patients with asymptomatic Brugada syndrome. Circ J
2009; 73: 580-583.

19. Antzelevitch C, Brugada P, Borggrefe M, Brugada J, Brugada R, Corrado D, et al. Brugada syndrome: Report of the second consensus conference. Heart Rhythm 2005; 2: 429-440.

20. Franz MR. Method and theory of monophasic action potential recording. Prog Cardiovasc Dis 1991; 33: 347-368.

21. Pak HN, Hong SJ, Hwang GS, Lee HS, Park SW, Ahn JC, et al. Spatial dispersion of action potential duration restitution kinetics is associated with induction of ventricular tachycardia/fibrillation in humans. J Cardiovasc Electrophysiol 2004; 15: 1357-1363.

22. Yamazaki M, Osaka T, Yokoyama E, Kodama I. A case of shortcoupled variant of torsades de pointes characterized by spatial heterogeneity of action potential duration and its restitution kinetics. J Interv Card Electrophysiol 2006; 17: 35-40.

23. Selvaraj RJ, Picton P, Nanthakumar K, Chauhan VS. Steeper restitution slopes across right ventricular endocardium in patients with cardiomyopathy at high risk of ventricular arrhythmias. Am J Physiol 2007; 292: H1262-H1268.

24. Park SW, Kim YH, Park SM, Rha SW, Ahn JC, Lim DS, et al. Relation between action potential restitution kinetics and inducibility of ventricular fibrillation in Brugada syndrome (abstract). Pacing Clin Electrophysiol 2003; 26: S24.

25. Bass BG. Restitution of the action potential in cat papillary muscle. Am J Physiol 1975; 228: $1717-1724$.

26. Veldkamp MW, Viswanathan PC, Bezzina C, Baartscheer A, Wilde AA, Balser JR. Two distinct congenital arrhythmias evoked by a multifunctional $\mathrm{Na}(+)$ channel. Circ Res 2000; 86: E91 -E97.

27. Wang DW, Makita N, Kitabatake A, Balser JR, George AL Jr. Enhanced $\mathrm{Na}(+)$ channel intermediate inactivation in Brugada syndrome. Circ Res 2000; 87: E37-E43.

28. Ashino S, Watanabe I, Kofune M, Okumura Y, Ohkubo K, Nakai $\mathrm{T}$, et al. Intravenous administration of quinidine gluconate prevents the induction of ventricular fibrillation in patients with Brugada syndrome. J Nihon Univ Med Assoc 2008; 67: 299-303.

29. Kimura M, Kobayashi T, Owada S, Ashikaga K, Higuma T, Sasaki $\mathrm{S}$, et al Mechanism of ST elevation and ventricular arrhythmias in an experimental Brugada syndrome model. Circulation 2004; 109: $1660-1666$

30. Ohkubo K, Watanabe I, Takagi Y, Okumura Y, Ashino S, Kofune $\mathrm{M}$, et al. Endocardial electrograms from the right ventricular outflow tract after induced ventricular fibrillation in patients with Brugada syndrome. Circ J 2007; 71: 1258-1262.

31. Morita H, Fukushima-Kusano K, Nagase S, Takenaka-Morita S, Nishi N, Kakishita M, et al. Site-specific arrhythmogenesis in patients with Brugada syndrome. J Cardiovasc Electrophysiol 2003; 14: $373-379$.

32. Coronel R, Casini S, Koopmann TT, Wilms-Schopman RA, Verkerk $\mathrm{AO}$, de Groot JR, et al. Right ventricular fibrosis and conduction delay in a patient with clinical signs of Brugada syndrome: A combined electrophysiological, genetic, histopathologic, and computational study. Circulation 2005; 112: 2769-2777.

33. Postema P, Dessel PF, de Bakker JM, Dekker LR, Linnenbank AC, Hoogendijk MG, et al. Slow and discontinuous conduction conspire in Brugada syndrome: A right ventricular mapping and stimulation study. Circ Arrhythmia Electrophysiol 2008; 1: 379-386.

34. Furushima H, Chinushi M, Iijima K, Izumi D, Hosaka Y, Aizawa Y. Significance of early onset and progressive increase of activation delay during premature stimulation in Brugada syndrome. Circ $J$ 2009; 73: $1408-1415$.

35. Coronel R, Wilms-Schopman FJ, Opthof T, Janse MJ. Dispersion of repolarization and arrhythmogenesis. Heart Rhythm 2009; 6: $537-543$.

36. Franz MR, Bargheer K, Rafflenbeul W, Haverich A, Lichtlen PR. Monophasic action potential mapping in human subjects with normal electrocardiograms: Direct evidence for the genesis of the T wave. Circulation 1987; 75: 379-385.

37. Koller BS, Karasik PE, Solomon AJ, Franz MR. Relation between repolarization and refractoriness during programmed electrical stimulation in the human right ventricle: Implications for ventricular tachycardia induction. Circulation 1995; 91: 2378-2384.

38. Ino T, Karagueuzian HS, Hong K, Meesmannn M, Mandel WJ, Peter T. Relation of monophasic action potential recorded with contact electrode to underlying transmembrane action potential properties in isolated cardiac tissues: A systemic microelectrode validation study. Cardiovasc Res 1988; 22: 255-264.

39. Yue AM, Franz MR, Roberts PR, Morgan JM. Global endocardial electrical restitution in human right and left ventricles determined by non-contact mapping. J Am Coll Cardiol 2005; 46: 1067-1075. 